\title{
On the diagnosis of climate sensitivity using observations of fluctuations
}

\author{
D. B. Kirk-Davidoff \\ Department of Atmospheric and Oceanic Science, University of Maryland, College Park, MD 20742, USA \\ Received: 13 May 2008 - Published in Atmos. Chem. Phys. Discuss.: 30 June 2008 \\ Revised: 9 January 2009 - Accepted: 9 January 2009 - Published: 2 February 2009
}

\begin{abstract}
It has been shown that lag-covariance based statistical measures, suggested by the Fluctuation Dissipation Theorem (FDT), may allow estimation of climate sensitivity in a climate model. Recently Schwartz (2007) has used measures of the decay of autocorrelation in a global surface temperature time series to estimate the real world climate sensitivity. Here we use a simple climate model, and analysis of archived coupled climate model output from the IPCC AR4 runs, for which the climate sensitivity is known, to test the utility of this approach. Our analysis of these archived model output data show that estimates of climate sensitivity derived from century-long time scales typically grossly underestimate the models' true climate sensitivity. We analyze the behavior of the simple model with adjustable heat capacity in two surface layers, subject to various stochastic forcings and for various climate sensitivities, modulated by albedo and water vapor feedbacks. We use our simple climate model to demonstrate:
\end{abstract}

1. that a much longer time series would be required to accurately diagnose the earth's climate sensitivity than is presently available

2. that for shorter time series there is a systematic bias towards underpredicting climate sensitivity,

3. that the addition of a second heat reservoir weakly coupled to the first greatly reduces the decorrelation timescale of short temperature time series produced by the model, aggravating the tendency to underestimate climate sensitivity, and

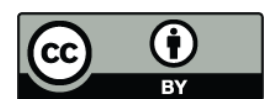

Correspondence to: D. B. Kirk-Davidoff (dankd@atmos.umd.edu)
4. that because of this it is possible to have a selection of models in which the climate sensitivity is inversely related to the decorrelation time scale, as is true for the IPCC models.

\section{Introduction}

An accurate determination of the earth's climate sensitivity, the expected mean surface temperature response to a doubling of carbon dioxide concentration, has been the outstanding problem in climate dynamics for the last several decades. Leith (1975) and Bell (1980) introduced the idea that the climate sensitivity of the earth or of a general circulation model (GCM) could be predicted using the Fluctuation Dissipation Theorem (FDT) (Callen and Green, 1952). The FDT states that for systems near equilibrium, the rate at which anomalies dissipate is related to the sensitivity of the system to a finite change in forcing. Bell (1980) and Cionni et al. (2004) were able to show that global climate sensitivity could in fact be predicted to useful accuracy for a simplified climate model and for a highly complex chemical GCM, respectively, using measures based on the FDT. Gritsun and Branstator (2007) showed that the complex patterns of a GCM response to spatially isolated heating could be predicted using the FDT, and noted that long time series $(\sim 100$ years $)$ were required for accurate diagnosis of the response matrix. In addition, they present a clear justification for the applicability of the FDT to models of the atmospheric flow, and thus potentially to the real climate system. Langen and Alexeev (2005) used the FDT to predict the zonal mean response of a GCM to climate forcing, using 500 years of model output data. North et al. (1993) and von Storch (2004) considered the dissipation of fluctuations in a GCM and discussed the applicability of

Published by Copernicus Publications on behalf of the European Geosciences Union. 
the FDT to climate models. Great hopes have been staked on the use of the FDT to diagnose sensitivity using satellite infrared radiance data (Goody et al., 1998).

In all of these discussions, two aspects of the potential use of the FDT for climate sensitivity diagnoses have been neglected. First, there has been no explicit discussion of the use of the FDT in the case where the heat capacity of the system is uncertain. Second, there has been no thorough investigation of how the length of the time series needed for an accurate diagnosis of climate sensitivity depends on the parameters (climate sensitivity, heat capacity, number of independent variables) of the system. In this paper, we investigate the practical usefulness of the FDT for the purpose of diagnosing climate sensitivity. To do so, we use an extremely simple climate model that is nevertheless includes parameters controlling climate sensitivity, heat capacity and internal heat transports.

Recently, Schwartz (2007) has used analysis of lagautocorrelation of the global mean surface temperature time series for the last 150 years, along with analysis of ocean heat storage over the last 50 years, to simultaneously calculate the earth's effective heat capacity and climate sensitivity. Schwartz (2007) diagnosed a value of the climate sensitivity $\left(0.3 \mathrm{~K} \mathrm{~W}^{-1} \mathrm{~m}^{2}\right)$ that was quite low compared to other estimates of climate sensitivity $\left(0.75 \mathrm{~K} \mathrm{~W}^{-1} \mathrm{~m}^{2}\right)$. Schwartz argued that the discrepancy was due to the models' overestimate of the climate system's decorrelation time scale, arguing that while his results showed a decorrelation time scale of about 5 years, climate models typically required some 30 years to fully adjust to a step change in climate forcing. We will show that actual climate model time series typically show decorrelation time scales similar to that found by Schwartz (2007) for observed global average surface air temperature. Thus, application of Schwartz's technique to climate model output results in a substantial underestimate of the climate models' climate sensitivity. We will use a simple heuristic climate model to show how a model with a relatively long adjustment time may nevertheless exhibit a short decorrelation time-scale.

Foster et al. (2008), Knutti et al. (2008), Scafetta (2008), and Schwartz (2008) also address the claims of Schwartz (2007). Knutti et al. (2008) compares predicted and realized climate sensitivity in the AR4 GCM runs, while Scafetta (2008) and Foster et al. (2008) use simplified a climate model to simulate the ability of the FDT to estimate climate sensitivity. They also concluded that the method of Schwartz (2007) would systematically underestimate climate sensitivity. The analysis of the impact of multiple heat capacities presented here is unique to this study.

In Sect. 2 we review the use of lag-correlation analysis to estimate climate sensitivity. In Sect. 3, we apply Schwartz (2007)'s method to the IPCC AR4 model runs, to test the method on modeled climate systems whose climate sensitivity is known. In Sects. 4 and 5 we introduce our simple climate model and apply it to explain the systematic under- estimate of climate sensitivity by the lag-correlation method. These errors are due both to the inadequate length of the time series involved, and to the assumption of a single heat capacity for the climate system.

\subsection{Fluctuation dissipation and climate sensitivity}

To gain an intuitive understanding of the use of lagcorrelation techniques to estimate climate sensitivity, the reader should imagine the climate system as a linear oscillator. By observing unforced oscillations of the temperature, we hope to estimate the response of the climate system to a step change in climate forcing. We assume that the unforced fluctuations, though in reality driven by internal instabilities, can be treated as though they were driven by random fluctuations of the forcing. The system tends to return to its equilibrium temperature because of the dominant blackbody feedback, whereby an increase in temperature leads to an increase in outgoing radiation, and thus a return to equilibrium. In a system with large climate sensitivity, a small fluctuation in the climate state leads to feedbacks that tend to reduce the response of outgoing radiation to the climate perturbation and slow the return of the system to equilibrium. On the other hand, in a system with small climate sensitivity, the same fluctuation in climate state causes feedbacks that tend to hasten the return to equilibrium. Thus a time series of surface temperature ought to show larger lag-autocorrelation in the system with larger climate sensitivity, and smaller lagautocorrelation in the system with smaller climate sensitivity.

There are a number of reasons to suspect that this relationship might not be so simple in the real world. The relative importance of external and internal drivers of global temperature is different at different time scales, and it is reasonable to suppose that feedbacks might therefore act differently at different time scales. For instance, interannual variations of global mean surface temperature in the real climate system are largely driven by internal oscillations of the system (e.g. ENSO), where the mean temperature change is a small residual of large and nearly compensating patterns of heating and cooling. In this case it is clear that any geographic dependence in the strength of climate feedbacks might make the global feedback have a different relationship to the global temperature for these internally forced variations than for externally forced climate change (e.g. by a secular trend in carbon dioxide concentration).

These concerns suggest that the FDT should be applied to the climate system using a large state vector, containing many climate relevant variables. Indeed Dymnikov and Gritsun (2005) and Majda et al. (2005) have shown that the FDT applies well to the fully non-linear systems represented by climate models, and Gritsun and Branstator (2007) have confirmed that the response of a GCM to a spatially constrained climate forcing can be predicted in detail using the FDT on a large state vector (approximately 1800 variables). Nevertheless, in this work we focus on the ability of the FDT to 
diagnose climate sensitivity using a single global temperature variable so that we can focus on the data requirements for this diagnosis, and especially on the role of an unknown heat capacity as an obstacle to accurate diagnosis.

We now formalize our treatment of the relationship between the lag-correlation and climate sensitivity for a purely linear system. Our discussion follows that of Penland and Sardeshmukh (1995) (a more general treatment, resulting in the same formula for predicting climate system response to forcing from the lag covariance matrix of the system variables, is given in Gritsun and Branstator, 2007). Consider a linear system forced by white noise $(\xi)$ and a fixed forcing $f$ :

$\frac{d \mathbf{x}}{d t}=\mathbf{B} \mathbf{x}+\underline{\xi}+f$

A change in $f$ from $f_{0}$ to $f_{1}$ will result in a change in the long-term mean value of $\mathbf{x}$ of $\left(\overline{\mathbf{x}}_{1}-\overline{\mathbf{x}}_{0}\right)=-\mathbf{B}^{-1}\left(f_{1}-f_{0}\right)^{-1}$. Thus we call $-\mathbf{B}$ the sensitivity matrix. $\mathbf{- B}$ can be determined by observation of the lag-covariances of the system, as follows:

$\mathbf{B}=\frac{1}{\tau} \ln \mathbf{C}_{x x}(\tau) \mathbf{C}(0)^{-1}$,

where $\mathbf{C}_{x x}(\tau)=<\mathbf{x}(t+\tau) \mathbf{x}^{T}(t)>$, and $\tau$ is an arbitrary time lag. By taking the exponent of both sides of Eq. 2, and then integrating over all time lags $\tau$, one obtains

$$
\int_{0}^{\infty} \exp (\tau \mathbf{B}) d \tau=\int_{0}^{\infty} \mathbf{C}_{x x}(\tau) \mathbf{C}(0)^{-1} d \tau
$$

or

$$
-\mathbf{B}^{-1}=\int_{0}^{\infty} \mathbf{C}_{x x}(\tau) \mathbf{C}(0)^{-1} d \tau
$$

which is a form of the FDT. Note that no use is made in this estimate of the white noise forcing, $\xi$, and that the sensitivity is given in units of time: it is the time scale of the equivalent restoring force of the system. Gritsun and Branstator (2007), following Dymnikov and Gritsun (2005) and Majda et al. (2005), have shown that the same result (i.e. that the equilibrium response of the system to a sustained small perturbation can be calculated using the integrated lag covariance of the unperturbed system) can be obtained for nonlinear systems as long as their probability density functions are approximately gaussian. In any practical application of the FDT to climate sensitivity diagnosis, we must be able to write the controlling equations of the climate system so that the climate forcing appears on the right hand side in units of Kelvins per unit time. This requires independent knowledge of the heat capacity of the climate system.

One can use either Eqs. 2 or 4 to obtain B. Schwartz (2007) used a scalar equivalent to Eq. 1, to estimate the decorrelation time scale for the time series of global mean temperature, $-\frac{1}{\tau} \ln r(\tau)$, where $r(\tau)$ is the autocorrelation of $x$ at lag $\tau$, by plotting its value versus $\tau$ for lags of up to 20 years, and looking for a tendency of the value of the decorrelation time scale to asymptote to a fixed value as $\tau$ increased. He combined this estimate with a separate estimate of global heat capacity to produce his estimate of climate sensitivity.

Cionni et al. (2004) employed a method inspired by the FDT, where instead of calculating the integral of the lag covariance matrix of the system $\mathbf{x}$, they calculated the integral of the lag covariance of the system $\mathbf{x}$ and the forcing $\underline{\xi}$ :

$$
-\mathbf{B}_{\mathrm{est}}{ }^{-1}=\int_{0}^{\tau_{L}} \mathbf{R}_{\mathbf{x} \xi}(\tau) d \tau
$$

where $\mathbf{B}_{\text {est }}$ is an estimate of the best-fitting linear operator $\mathbf{B}$, whose accurracy is limited by the finite length of the time series $\mathbf{x}$, and where

$\mathbf{R}_{x \xi}(\tau)=\int_{0}^{\infty} \mathbf{x}(t+\tau) \underline{\xi}(t)^{T} d t / \int_{0}^{\infty} \underline{\xi}(t) \underline{\xi}(t)^{T} d t$.

$\tau_{L}$, the upper limit of integration in Eq. 5 is the lag beyond which no significant correlation is observed. One way to choose a value for this variable is to integrate Eq. 5 for a sufficiently large $\tau_{L}$ that $\mathbf{B}_{\text {est }}$ reaches a maximum, and then begins to decrease. Cionni et al. (2004) accurately estimated climate sensitivity based on a 10 year time series of model output data from a GCM run with fixed sea surface temperature and known forcings. In their study, diagnosis of effective heat capacity was not needed because only the small land surface heat capacity contributed to the decorrelation time. In the real world the task becomes more difficult, due to short time series and a lack of accurate forcing information.

\section{Application to IPCC AR4 model output}

Here we apply the tools described above to test the ability of analysis based on lag-correlation of temperature timeseries, combined with estimation of global heat capacity by the methods of Schwartz (2007) to predict climate sensitivity in the IPCC AR4 model runs. Figure 1a shows the autocorrelation of the detrended global mean surface air temperature as function of lag (in years) for 18 coupled model simulations of the 20 th century (the " $20 \mathrm{c} 3 \mathrm{~m}$ " runs). The curve for the GISS global mean surface air temperature (Hansen et al., 1996) is shown in light blue, and is the same curve shown in Schwartz (2007), Fig. 2c. Note the good agreement between the GISS curve and the IPCC model curves. Figure 1b shows the decorrelation time scale, $-\frac{1}{\tau} \ln r(\tau)$ for the same global mean surface air temperature time series. Again, the light blue curve showing the GISS observations falls well within the range of the IPCC model lag autocorrelation curves. The variability of the decorrelation time scale as a function of lag for each model, and for the observed data are due to a combination of random error associated with the relatively short time series involved, and deviations from linearity of the models. In the absence of much longer runs for each model, we cannot distinguish the role of each. We obtain our 
(a)

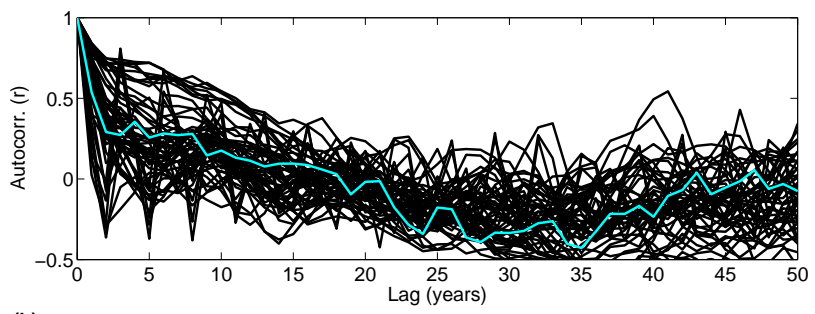

(b)

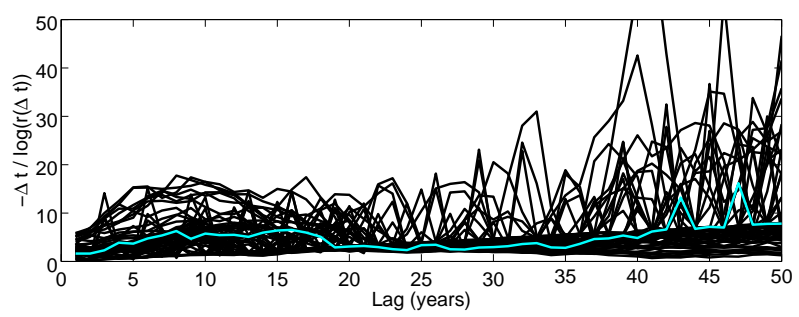

Fig. 1. (a) Lag autocorrelation of global mean temperatures from the GISS surface air temperature record (light blue), and from the IPCC AR4 20th Century runs (black). (b) De-correlation timescale $\tau / \ln (r(\tau)))$ for the time global mean time series, and the same color-coding.

best estimate of the model decorrelation time scale by finding the lag for which the autocorrelation is less than zero, and averaging the decorrelation time scale either over all lags up to the lag with zero correlation, or over the three years prior to that lag, whichever is greater. Using this method, the average decorrelation time scale for the models is $8.3 \mathrm{yr}$, with a standard deviation of $4.5 \mathrm{yr}$, and a range of $2.8-18.5 \mathrm{yr}$. Thus, Schwartz (2007)'s estimate of $5 \mathrm{yr}$ falls in the range of the model decorrelation time scales.

Following Schwartz (2007) we calculate the models' effective heat capacity using two methods: by regressing the global heat storage against the global temperature, and by taking the ratio of the trends of heat storage and temperature. Heat storage is obtained by integrating the global downward flux of heat at the atmosphere's lower boundary, which is the sum of the latent and sensible heat fluxes, and the net downward long and shortwave fluxes. Scatter plots of annual and global mean heat storage anomaly versus annual and global mean temperature anomaly are shown in Fig. 2, offset from zero mean for clarity. The light blue points refer the observed ocean heat storage, and were taken by digitizing the lower right panel of Fig. 4 in Schwartz (2007). That figure plots GISS surface temperatures against ocean heat storage from the surface to $3000 \mathrm{~m}$ from the Levitus data set (Levitus et al., 2005), scaled upwards to account for the heat capacity of land. As has been pointed out by Forest et al. (2006), it is apparent that the slope of the dependence of heat storage on surface temperature (shown in light blue) for the observed data is small $\left(12 \mathrm{~W} \mathrm{yr} \mathrm{m}^{-2}\right)$ compared to the range of values found for the models. The average slope for the model data is $90 \mathrm{~W} \mathrm{yr} \mathrm{m} \mathrm{K}^{-1}$, with a minimum of $10 \mathrm{~W} \mathrm{yr} \mathrm{m}{ }^{-2} \mathrm{~K}^{-1}$,

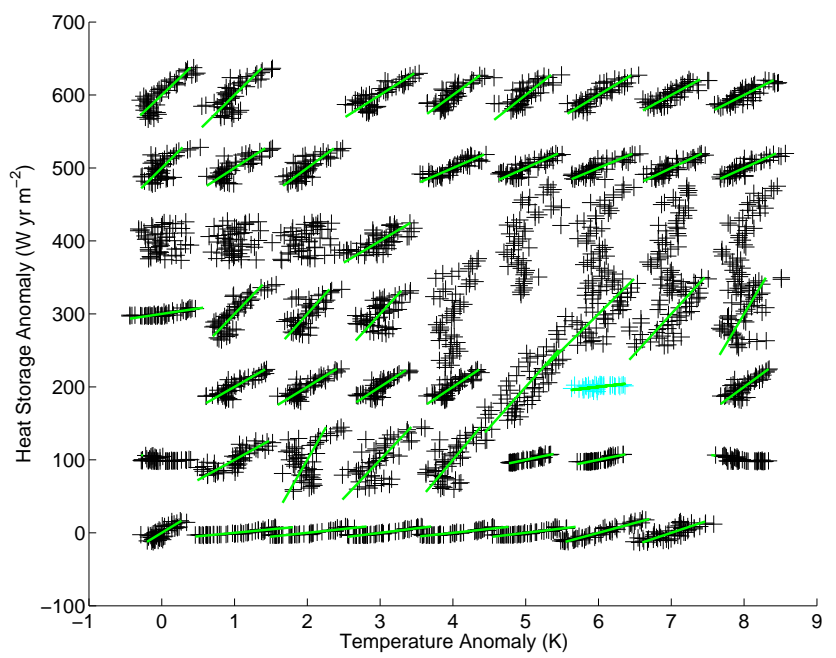

Fig. 2. Integrated net downward surface heat flux (in units of $\mathrm{W} \mathrm{yrm}^{-2}$ ) plotted versus global mean surface air temperature anomaly. IPCC Models output data are shown in black as in Fig. 1, with the GISS temperature and Levitus et al. (2005) heat storage figures taken by digitizing Fig. 4 of Schwartz (2007), shown in light blue.

and a standard deviation of $81 \mathrm{~W} \mathrm{yr} \mathrm{m} \mathrm{m}^{-2} \mathrm{~K}^{-1}$.

With these two quantities calculated, we can now, following Schwartz (2007), make an estimate of the equilibrium climate sensitivity, and see how well it predicts the actual model sensitivity. One difficulty with this procedure concerns the definition of climate sensitivity. Since the theory underlying the use of the decorrelation time scale to predict climate sensitivity assumes small perturbations to the climate state, the estimate ought to work best for a small climate forcing perturbation, such that state-dependent feedbacks are functioning near the unperturbed initial state. However, for reasons of practical interest, climate models are generally run for finite periods using finite forcing. For the IPCC AR4 coupled model runs, we can define climate sensitivity as either:

1. the full response of global mean surface air temperature to a doubling of carbon dioxide, where the doubling occurs over a period of 150 years, and the models are then run for an additional 100 years allowing an approximate equilibration,

2. as the transient response of global mean surface air temperature during a 1 percent per year increase of carbon dioxide, or

3 . as the transient response of global mean surface air temperature to one of several particular emission scenarios in which a range of greenhouse gases increase monotonically over the course of the model runs.

In Fig. 3, we show that these sensitivities are related but different. Here, transient sensitivity of the models (in $\mathrm{K} / 100 \mathrm{yr}$ ) derived from a linear fit to the trend of global 


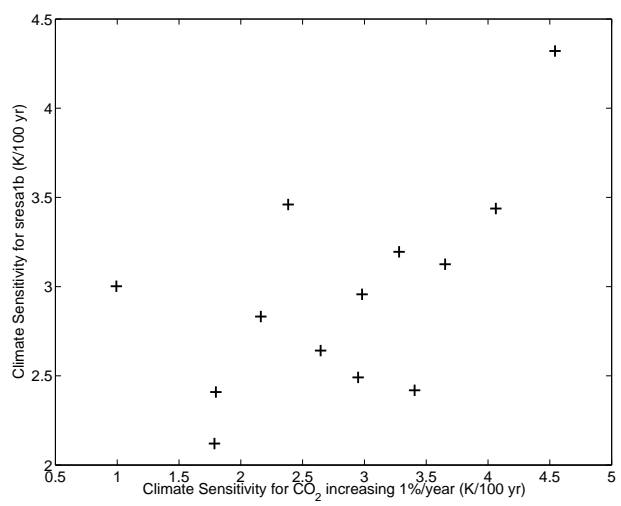

Fig. 3. Climate sensitivity from a linear fit to the first 50 years of the IPCC AR4 runs with $1 \%$ per year increasing $\mathrm{CO} 2$ versus climate sensitivity derived from the temperature difference between the years 2100 and 2100 in the SRESalb runs.

mean surface air temperature for the first 50 years of runs with carbon dioxide increasing $1 \% \mathrm{yr}^{-1}$ is compared with climate sensitivity derived from the temperature difference for the years 2000 to 2100 in the "SRESalb" climate forcing scenario. Among the nineteen models with output for both experiments stored in the PCMDI archive, the correlation coefficient of these two measures of climate sensitivity is 0.57 , indicating that important aspects of the climate system (whether the effective climate forcing due to the imposed changes in greenhouse gas forcing, the climate feedbacks or the effective heat capacities of different components of the climate system) respond differently to these two forcing scenarios. In the following discussion we will present results comparing the climate sensitivity derived using the procedure of Schwartz (2007) with the equilibrium climate response of the models in the $1 \% \mathrm{yr}^{-1}$ forcing scenario. The reader should note however, that similar comparisons have been done with the SRESa1b-derived sensitivities, with broadly similar results (not shown).

In Fig. 4a, we confirm that the estimates of global effective heat capacity derived from regression of global heat storage against global heat temperature in the $20 \mathrm{c} 3 \mathrm{~m}$ model runs are reasonably well correlated $(r=0.70)$ with the estimates derived from the ratio of the trends of the two numbers. Heat capacity is shown in units of equivalent mixed layer depth $(\mathrm{m})$, calculated by dividing the heat storage per unit area by the heat capacity of $1 \mathrm{~m}^{3}$ of sea water. Having established this, we proceed using the average of the two methods. Figure $4 \mathrm{~b}$ shows the relationship between the decorrelation time scale and the effective global heat capacity. Contrary to the prediction of a linear, single heat reservoir theory of climate dynamics, the relationship is weak, and anticorrelated $(r=-0.48)$ : models with large decorrelation time scales tend to have small heat storage. When we compare decorrelation time with model sensitivity derived from the mean difference between the first and last yen years of the IPCC AR4 (a)

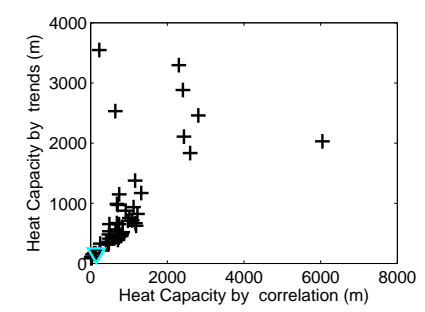

(c)

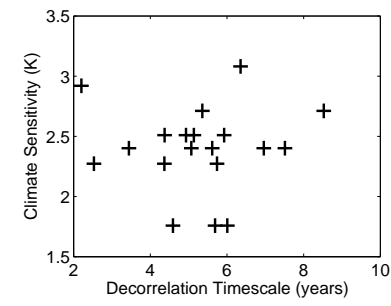

(b)
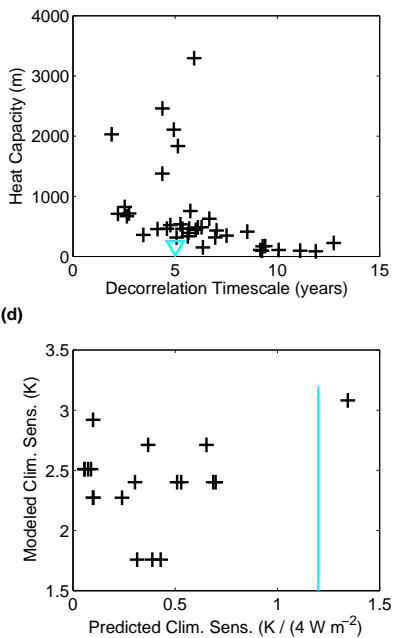

Fig. 4. (a) Scatter plot of heat capacity (in units of meters of sea water) calculated for the IPCC AR4 twentieth century (20c3m) model runs by direct correlation of heat storage and temperature versus heat capacity calculated by the ratio of the trends of heat storage and temperature over the 20th century. (b) Heat capacity from the mean of the two methods used in (a) versus decorrelation time scale. (c) Climate sensitivity derived from the mean difference of the first and last ten years of the AR4 runs forced with a 1 percent per year increase in $\mathrm{CO}_{2}$ versus decorrelation time scale. (d) Climate sensitivity predicted by the method of Schwartz (2007) from the IPCC AR4 20th century runs, versus actual climate sensitivity derived from the 1 percent per year to doubling of $\mathrm{CO}_{2}$ runs. Schwartz's (2007) estimate of climate sensitivity is indicated with the light blue line.

runs with 1 percent per year increases of $\mathrm{CO}_{2}$ (Fig. 4c), the correlation is very weak and $(r=-0.05)$. Finally we compare this "true" climate model climate sensitivity with the estimate of climate sensitivity calculated by dividing the decorrelation timescale by the effective heat global heat capacity in the 20th century runs, as calculated by Schwartz (2007). We find that the estimate of climate sensitivity made by Schwartz $\left(0.30 \mathrm{~K} \mathrm{~W}^{-1} \mathrm{~m}^{2}\right)$ fall squarely within the range of climate sensitivity derived from the IPCC AR4 models (mean $=0.22 \mathrm{~K} \mathrm{~W}^{-1} \mathrm{~m}^{2}$, $\min =9.9 \times 10^{-4} \mathrm{~K} \mathrm{~W}^{-1} \mathrm{~m}^{2}$, $\max =1.8 \mathrm{~K} \mathrm{~W}^{-1} \mathrm{~m}^{2}$. When the estimated climate sensitivity is compared with the actual climate sensitivity derived from the equilibrated $1 \%$ per year increasing $\mathrm{CO}_{2}$ runs (Fig. 4 d), the relationship is, again, weak $(r=0.27)$.

These results show that contra Schwartz (2007), the decorrelation time scale of the global mean temperature time series is not in conflict with the range of model estimates, but that the global effective heat capacity is overestimated by the great majority of the IPCC AR4 models. Since climate sensitivity is predicted to be equal to the decorrelation time scale divided by the ocean heat uptake, excessive heat uptake combined with a realistic decorrelation timescale should lead the models to underestimate climate sensitivity. However, the fact that the models themselves do not obey this relationship 
(i.e. the ratio of decorrelation time scale over effective heat capacity is poorly correlated with model climate sensitivity) is a strong argument against the use of a single-variable version of the FDT to estimate the earth's climate sensitivity. In the next two sections we use a very simple model of the climate system to explore why the theory might fail for climate system models, and for the real climate system.

\section{Model description}

For simplicity and ease of integration, we make use of a global mean climate model with a single atmospheric layer and purely radiative interactions between the surface and the atmosphere. The features needed include rapid integration, an ability to simulate arbitrary climate sensitivity, and simulation of both radiative and dynamical energy fluxes. The model includes two ocean mixed layers (upper and lower) with an adjustable linear coupling, to simulate heat storage in the ocean, a single atmospheric layer to account for greenhouse warming, and gray-body radiation. Feedbacks include a temperature-dependent atmospheric infrared emissivity (intended to represent the water vapor feedback), and a temperature-dependent surface albedo (intended to represent ice-albedo and low cloud feedbacks). The model is forced with modified red noise applied to either the solar variability or the infrared emissivity.

The thermodynamic equations for the lower and upper mixed layers and the atmosphere are:

$$
\begin{aligned}
& C_{s 2} \frac{d T_{s 2}}{d t}=-\gamma\left(T_{s}-T_{s 2}\right) \\
& C_{s} \frac{d T_{s}}{d t}=-\gamma\left(T_{s 2}-T_{s}\right)+\frac{1}{4} S\left(1-\epsilon_{S}\right)(1-\alpha)-\sigma T_{s}^{4}+\epsilon \sigma T_{a}^{4} \\
& C_{a} \frac{d T_{a}}{d t}=\frac{1}{4} \epsilon_{S} S(1+\alpha)+\epsilon \sigma T_{s}^{4}-2 \epsilon \sigma T_{a}^{4}
\end{aligned}
$$

where $C_{s}$ and $C_{s 2}$ are the heat capacities of the upper and lower mixed layers, $T_{s}$ and $T_{s 2}$ are their respective temperatures, $C_{a}$ and $T_{a}$ are the heat capacity and temperature of the atmosphere, $\gamma$ is the coupling time constant between the upper and lower mixed layers, $S$ is the solar constant, $\epsilon_{S}$ is the solar absorption of the atmosphere, $\alpha$ is the surface albedo, $\sigma$ is the Stefan-Boltzmann constant, and $\epsilon$ is the infrared emissivity of the atmosphere.

The atmospheric emissivity and the surface albedo are subject to linear feedbacks on atmospheric and surface temperature, respectively:

$$
\epsilon=\epsilon_{0}+f_{\epsilon}\left(T_{a}-T_{\epsilon}\right)+c_{\epsilon} A_{\epsilon}
$$

$\alpha=\alpha_{0}+f_{\alpha}\left(T_{s}-T_{\alpha}\right)$

where $\epsilon_{0}$ and $\alpha_{0}$ are the basic state emissivity and surface albedo, respectively, $T_{\epsilon}$ and $T_{\alpha}$ are the basic state atmosphere and surface temperatures, respectively, and $f_{\epsilon}$ and $f_{\alpha}$ are the feedback parameters. $A_{\epsilon}$ is a red-noise forcing, and $c_{\alpha}$ is a constant that controls its magnitude. The solar constant can also be forced with red noise:

$S=S_{0}+c_{S} A_{S}$,

where $c_{S}$ is a constant controlling the magnitude of the forcing. $A_{\epsilon}$ and $A_{S}$ are generated using:

$$
\begin{aligned}
x_{i+1} & =\beta x_{i}+r \\
A & =\left[x-\frac{1}{2(1-\beta)}\right] \sqrt{1-\beta^{2}}
\end{aligned}
$$

where $r$ is a random variable with a flat distribution over the range [0 1], $\beta$ is the parameter that determines the autocorrelation of the red-noise forcing, and the scaling is performed after $x$ has been calculated for as many time steps as necessary. The autocorrelation of the resulting temperature time series will depend somewhat on the autocorrelation of the forcing, however, we hold the autocorrelation of the forcing fixed at 0.6 for a lag of one year for all the simulations discussed here, so that the autocorrelation of the modeled temperature variations changes only due to changes in heat capacity, climate sensitivity, and the coupling of the two heat reservoirs. The applicability of the FDT to this model is demonstrated below.

Solving Eqs. 7-9 for equilibrium conditions, with feedbacks and stochastic forcing assumed to be zero yields

$$
\begin{aligned}
T_{s} & =\sqrt[4]{\frac{S_{0}\left[1-\alpha+\frac{\epsilon_{S}}{2}\left(3 \alpha-1-\epsilon_{S} \alpha\right)\right]}{\sigma\left(1-\frac{\epsilon}{2}\right)}} \\
T_{a} & =\sqrt[4]{\frac{1}{2} T_{S}^{4}+\epsilon_{S} \frac{S_{0}}{4} \frac{1+\alpha-\epsilon_{S} \alpha}{2 \epsilon \sigma}}
\end{aligned}
$$

Figure 5a shows the equilibrium climate sensitivity of the surface temperature with respect to a change in the solar constant $S_{0}$ from $1360 \mathrm{~W} \mathrm{~m}^{-2}$ to $1370 \mathrm{~W} \mathrm{~m}^{-2}$ for a range of feedback parameter settings. For this simple model there is no ambiguity about "equilibrium", since the model can be run until energy balance is achieved to within $0.01 \mathrm{~W} \mathrm{~m}^{-2}$. Depending on the values of the parameter settings, the climate sensitivity for this forcing varies from 0.04 to $1.6 \mathrm{~K} \mathrm{~W}^{-1} \mathrm{~m}^{2}$, where the surface temperature has been divided by the change in solar forcing adjusted for albedo and geometry, $\frac{\Delta S}{4}(1-\alpha)$.

Figure $5 b$ shows that as the feedback parameters increase, the time required for the temperature to reach $90 \%$ of its equilibrium value also increases. Figure $5 \mathrm{c}$ confirms that, as predicted by the FDT, the inverse log of the lag autocorrelation $(-1 / \ln r(1 \mathrm{yr}))$ increases with increasing climate sensitvity. The autocorrelations are calculated for runs of 1600 years, with an ocean heat capacity equivalent to a mixed layer depth of $25 \mathrm{~m}$. All three of these variables - the adjustment time scale, the climate sensitivity, and the inverse log lag-autocorrelation - are correlated with one another with $r=0.98$. However, as shown in Fig. $5 d$, the ratio of the adjustment time to the climate sensitivity is not constant, but 

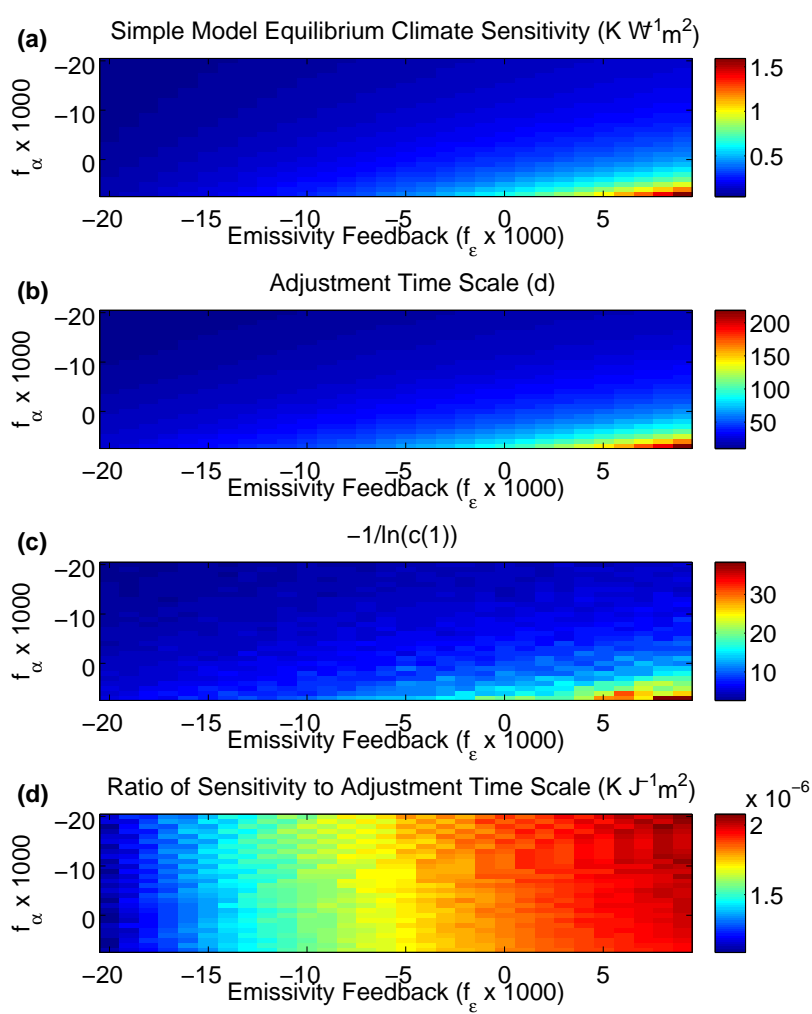

Fig. 5. (a) Equilibrium climate sensitivity of the simple climate model for a range of values of the feedback parameters $f_{\epsilon}$ and $f_{\alpha}$. (b) Adjustment timescale (time to $90 \%$ of response) for the same feedbacks. (c) $-1 / \ln r(1 \mathrm{yr})$ for the model surface temperature time series. (d) Ratio of climate sensitivity to adjustment time scale.

increases with increasing emissivity feedback, indicating a feedback-dependent bias in the relationship between the climate sensitivity and the adjustment timescale. This bias is due to the fact that the emissivity feedback parameter acts on $T_{a}$, rather than on $T_{s}$. For a lag-autocorrelation based methodology to accurately predict the climate sensitivity, the fluctuations of both $T_{a}$ and $T_{s}$ would have to be observed. Nevertheless, for a modest range of possible climate sensitivities (e.g. over a factor of 3), the ratio of the climate sensitivity to the adjustment time varies only by about $20 \%$. The more serious obstacles to the use of lag-autocorrelations to predict sensitivity arise from the possibility of multiple heat reservoirs, as we discuss in the next section.

\section{Simple model results and sensitivity experiments}

We now turn to the results of a series of experiments designed to show how statistics like those used by Schwartz (2007) can give misleading results about climate sensitivity. Figure 6 shows the simple model's response to changes in the solar constant. In Fig. 6a, the solar constant is increased by step change of $10 \mathrm{~W} \mathrm{~m}^{-2}$. The feedback parameters are (a)

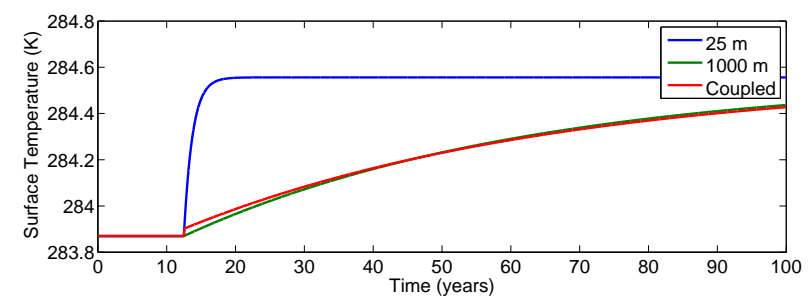

(b)

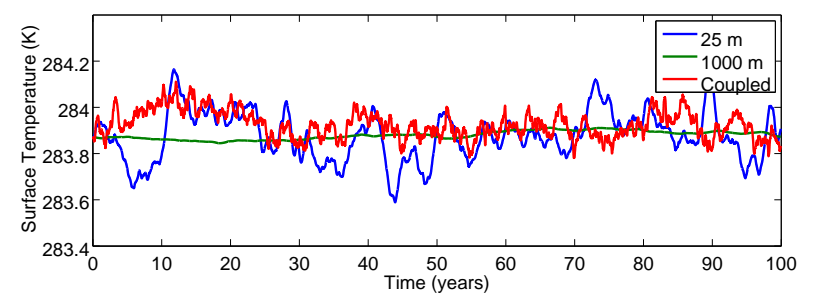

Fig. 6. (a) Response of the simple climate model to an instantaneous increase in the model solar constant for three different cases: a single $25 \mathrm{~m}$ ocean mixed layer (blue), a single $1000 \mathrm{~m}$ ocean mixed layer (green) and a $25 \mathrm{~m}$ ocean mixed layer coupled to $1000 \mathrm{~m}$ deeper mixed layer (red). (b) Response of the same model, with the same color-coded configurations, to random fluctuations in the solar forcing.

set to $f_{\epsilon}=0.002 \mathrm{~K}^{-1}$ and $f_{\alpha}=0.002 \mathrm{~K}^{-1}$. Three settings for heat capacity are compared: a single ocean mixed layer $25 \mathrm{~m}$ deep, a single layer $1000 \mathrm{~m}$ deep, and an upper layer of $25 \mathrm{~m}$ coupled to a lower layer of $1000 \mathrm{~m}$, with a coupling constant of $50 \mathrm{~W} \mathrm{~m}^{-2} \mathrm{~K}^{-1} \mathrm{~s}^{-1}$. Note that the adjustment time scale of the coupled case is identical to that of the $1000 \mathrm{~m}$ mixed layer case, except that there is an initial rapid approach to equilibrium in the time scale of the upper $25 \mathrm{~m}$ layer before the coupling between the layers acts to move the heat to the lower layer. In Fig. 6b, the solar constant varies randomly for the same parameter settings. The rapid oscillations of the coupled case indicates that the shallow upper mixed layer controls the time scale of the response. This behavior suggests that a diagnostic method based on decorrelation time scale will have trouble distinguishing between a system with low heat capacity on short time scales and one with low heat capacity on all time scales.

Pursuing this thread of argument, we now apply Schwartz (2007)'s methodology to much longer synthetic time series, in order to test its ability to extract the model's true dynamics from finite time series. Figure 7 shows the lag autocorrelation $r(\Delta t)$ for two model parameter settings. Figure $7 \mathrm{a}$ shows results for a single $25 \mathrm{~m}$ mixed layer. Figure $7 \mathrm{~b}$ shows results for a $25 \mathrm{~m}$ mixed upper layer, and a $1000 \mathrm{~m}$ mixed lower layer. In each case, the model was run for 10000 years, forced by white noise solar variability, and the time series of annual mean $T_{s}$ was calculated. Red curves show $r(\Delta t)$ calculated separately for 100100 -year chunks of data. The black curve shows $r(\Delta t)$ calculated for the full 10000 year record. These results demonstrate two key points. 
(a)

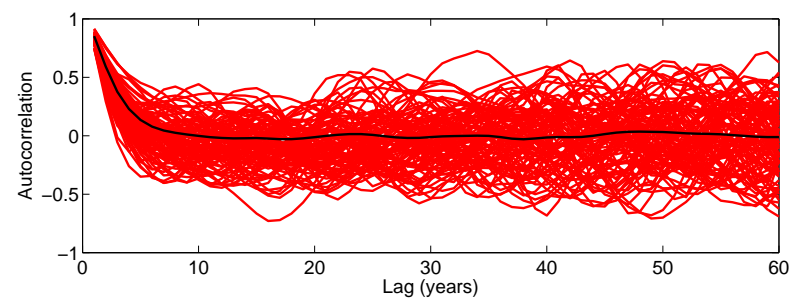

(b)

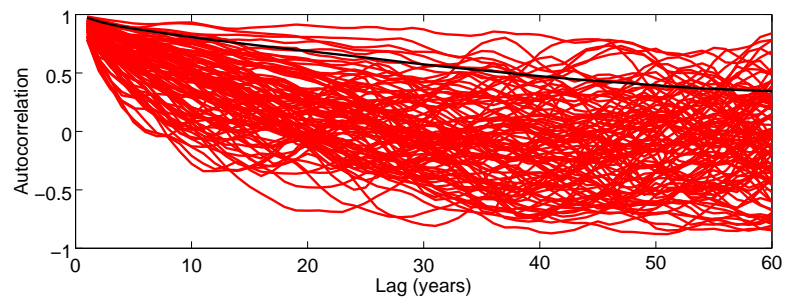

Fig. 7. (a) Lag autocorrelation for a ten thousand-year run of the simple model with a $25 \mathrm{~m}$ ocean mixed layer (black) and for a hundred hundred-year subsamples of the same run (red). (b) As for (a), for run with a $25 \mathrm{~m}$ ocean mixed layer coupled with a $1000 \mathrm{~m}$ lower mixed layer.

First, the uncertainty with which one can establish the value of the autocorrelation of a given 100 year time series is not the relevant uncertainty for the calculation of climate sensitivity. It is clear from panel Fig. 7a that a single curve of autocorrelation versus lag derived from a 100 year time series can give only a poor estimate of the true curve. For instance, while the autocorrelation curve derived from the 10000 year time series crosses zero at 10 years lag, the mean of the 100 year autocorrelation curves' zero crossing is 8.4 years, with a standard deviation of 5.7 years. Even for a model with a single heat capacity, analysis of the lag-autocorrelation of hundred year temperature time series results in both a large negative bias in estimating the true decorrelation time scale $(-\Delta t / \ln (r(\tau)$, averaged over lags from 8 to 12 years), and large variance of the decorrelation time scale of randomly selected hundred year segments. Thus, our simple model run with a single heat capacity, and with parameters yielding real decorrelation time scales ranging from $5 \mathrm{yr}$ up to $25 \mathrm{yr}$, produces 100 year segments within a standard deviation of the mean that have decorrelation time scales of $5 \mathrm{yr}$ or less. In particular, for a $25 \mathrm{yr}$ decorrelation time scale, the expected mean decorrelation time scale of a randomly selected $100 \mathrm{yr}$ segment is $15 \mathrm{yr}$, with a standard deviation of $10 \mathrm{yr}$. Thus, the fact that the observed temperature time series of the last $50 \mathrm{yr}$ of global temperature exhibits a decorrelation time scale of $5 \mathrm{yr}$ (Schwartz, 2007), means only that the true value could be anywhere from $3 \mathrm{yr}$ to $25 \mathrm{yr}$, and have the observed value within a single standard deviation of the expected value.

Second, when the model has more than a single heat reservoir, autocorrelations based on a hundred year sample can substantially underestimate both the true decorrelation time scale and the adjustment timescale. This is clear from panel (a)

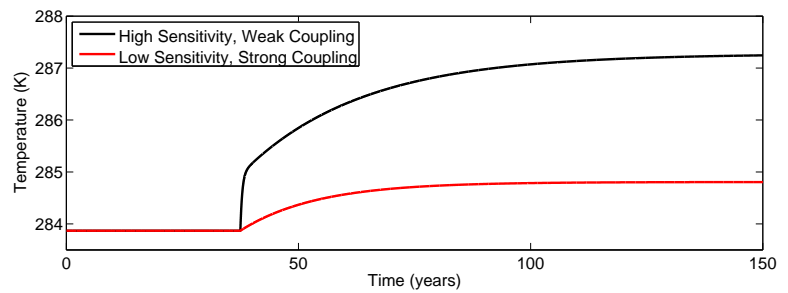

(b)

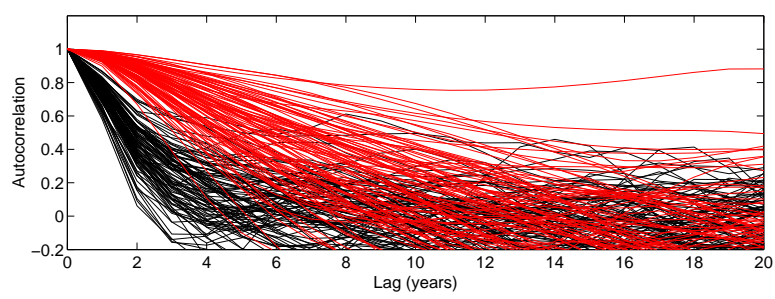

Fig. 8. (a) Response of the simple climate model to an instantaneous increase in the model solar constant for two different cases, one with low climate sensitivity and a single $1000 \mathrm{~m}$ ocean mixed layer, and one with high climate sensitivity and a $25 \mathrm{~m}$ mixed layer coupled to a $333 \mathrm{~m}$ deep mixed layer. (b) Lag autocorrelations of the surface temperature of the same two models, for 100100 -year runs each.

Fig. $7 \mathrm{~b}$ where the lag autocorrelation curve based on 10000 years of data exceeds $95 \%$ of the curves based on 100 year segments out to 30 years lag.

Finally we demonstrate how our simple model with a fast surface heat reservoir coupled to a large deep heat reservoir can fool the method of Schwartz (2007) into falsely ranking the climate sensitivities of the model run for various parameter choices. In Fig. 8, results in black are for parameter choices that give high climate sensitivity $\left(f_{\alpha}=f_{\epsilon}=0.002\right.$, Sensitivity: $0.4 \mathrm{~K} \mathrm{~W}^{-1} \mathrm{~m}^{2}$ ) and relatively weak coupling between the two mixed layers $\left(C_{s}=1 \times 10^{8} \mathrm{~J} \mathrm{~m}^{-2} \mathrm{~K}^{-1}\right.$ $(25 \mathrm{~m}), C_{s 2}=1.3 \times 10^{9} \mathrm{~J} \mathrm{~m}^{-2} \mathrm{~K}^{-1}(333 \mathrm{~m}), \gamma=5 \mathrm{~W} \mathrm{~m}^{-2}$ $\mathrm{K}^{-1}$ ). Results in red are for parameter choices that give low climate sensitivity $\left(f_{\alpha}=f_{\epsilon}=-0.01\right.$, Sensitivity: $\quad 0.1 \mathrm{~K} \mathrm{~W}^{-1} \mathrm{~m}^{2}$ ) and a single deep mixed layer $\left(C_{s}=1.3 \times 10^{9} \mathrm{~J} \mathrm{~m}^{-2} \mathrm{~K}^{-1}(333 \mathrm{~m}), \gamma=0 \mathrm{~W} \mathrm{~m}^{-2} \mathrm{~K}^{-1}\right)$.

Panel $a$ shows the response of each model to a step change in climate forcing. The high sensitivity, weakly coupled model jumps up rapidly, but after the temperature difference between the upper and lower mixed layers increases, the flux of heat from the upper to the lower layer slows the temperature rise until equilibrium is reached. The low sensitivity, single mixed layer model approaches equilibrium at the same constant e-folding rate. Panel $b$ shows the lag autocorrelation curves for 100 100-year segments of model output forced by noise. The lag autocorrelation curves show higher autocorrelation for the low climate sensitivity model, just the opposite of the result predicted by Schwartz (2007), even though the climate sensitivities vary by a factor of 4 . Thus, the low heat capacity of the surface layer, which would quite irrelevant to 
the response of the model to slowly increasing climate forcing, tricks the analysis method into predicting a small decorrelation time scale, and a small climate sensitivity, because of the short length of the observed time series. Only with a longer time series would the long memory of the system be revealed.

\section{Discussion and conclusions}

Some clear conclusions may be drawn from our work.

1. Although methods based on the FDT can produce precise, accurate estimates, given sufficient time, the time required for accurate evaluation of model sensitivity is in general several times longer than a model would require to come to equilibrium with a step-change in forcing.

2. Estimates of climate sensitivity using real global mean temperature variations are consistent with those using output data from the 20c3m IPCC AR4 model runs. There is no inconsistency between the model climate sensitivities and the observed global mean temperature decorrelation timescales, as claimed by Schwartz (2007).

3. Analysis of output from a simple climate model shows that the existence of multiple heat capacities in a climate system introduces systematic errors to the estimation of decorrelation time scale from the temperature time series. This may largely account for the systematic underestimate of IPCC model climate sensitivity by the lag-correlation method.

4. The large variability of the estimated climate sensitivity derived from these runs, even for multiple runs of the same model, confirms our prediction that estimation of climate sensitivity from a time series of a single variable requires much longer climate records than exist for the real world.

There is some prospect that improved diagnostic techniques based on the FDT may allow accurate estimation of climate sensitivity from climate fluctuations. Gritsun (2008) points out that an estimate of a more complete response vector of the climate system, obtained by analyzing a much larger state vector, might give better results. Alternatively, better results might be obtained using a different method to deal with the non-stationarity of the observed and modeled time series, for instance by concentrating on shorter time scale fluctuations during the winter season.
Acknowledgements. The author gratefully acknowledges helpful conversations with John Dykema, Andy Majda, and Raymond Najjar, and very thoughtful and thorough reviews of the original manuscript by Andrey Gritsun and Jin-Song von Storch. ECMWF ERA-40 data used in this study have been obtained from the ECMWF data server. We acknowledge the modeling groups for providing their data for analysis, the Program for Climate Model Diagnosis and Intercomparison (PCMDI) for collecting and archiving the model output, and the JSC/CLIVAR Working Group on Coupled Modelling (WGCM) for organizing the model data analysis activity. The IPCC Data Archive is supported by the Office of Science, US Department of Energy. The author's work on this subject was partially supported by a grant (G074756) from the University of Wisconsin.

Edited by: P. Haynes

\section{References}

Abramov, R. and A. Majda: Blended response algorithms for linear uctuation-dissipation for complex nonlinear dynamical systems, Nonlinearity, 20, 2793-2821, 2007.

Bell, T. L.: Climate sensitivity from fluctuation dissipation: some simple model tests, J. Atmos. Sci., 37, 1700-1707, 1980.

Callen, H. B. and Greene, R. F.: On a theorem of irreversible thermodynamics, Phys. Rev., 86, 702-710, 1952.

Cionni, I., Visconti, G., and Sassi, F.: Fluctuation dissipation theorem in a general circulation model, Geophys. Res. Lett., 31, L09206, doi:10.1029/2004GL019739, 2004.

Dymnikov, V. and Gritsun, A.: Current problems in the mathematical theory of climate, Izv. Acad. Sci. USSR, Atmos. Oceanic Phys., 41, 294-314, 2005.

Forest, C. E., Stone, P. H., and Sokolov, A. P.: Estimated PDFs of climate system properties including natural and anthropogenic forcings, Geophys. Res. Lett., 33, L01705, doi:10.1029/2005GL023977, 2006.

Foster G., Annan J. D., Schmidt G. A. and Mann, M. E.: Comment on "Heat Capacity, Time Constant, and Sensitivity of Earth Climate System" by S. Schwartz, J. Geophys. Res., 113, D15102, doi:10.1029/2007/JD009373, 2008.

Goody, R. M., Anderson, J. G., North, G. R.: Testing climate models: an approach, Bull. Am. Met. Soc., 79, 2541-2549, 1998.

Gritsun, A.: Interactive comment on "On the diagnosis of climate sensitivity using observations of fluctuations" by D. KirkDavidoff, Atmos. Chem. Phys. Discuss., 8, S5939-S5944, 2008.

Gritsun, A. and Branstator, G.: Climate response using a threedimensional operator based on the uctuation-dissipation theorem, J. Atmos. Sci., 64, 2558-2575, 2007.

Gritsun, A., Branstator, G., and Dymnikov, V.: Construction of the linear response operator of an atmospheric general circulation model to small external forcing, Num. Anal. Math. Modeling, 17, 399-416, 2002.

Gritsun, A. and Dymnikov, V.: Barotropic atmosphere response to small external actions, theory and numerical experiments, Atmos. Ocean Phys., 35(5), 511-525, 1999.

Hansen, J., Ruedy, R., Sato, M., and Reynolds, R.: Global surface air temperature in 1995: Return to pre-Pinatubo level, Geophys. Res. Lett., 23, 1665-1668, 1996, updated at: http://data. giss.nasa.gov/gistemp/. 
Knutti, R., Krhenmann, S., Frame, D. J., and Allen, M. R.: Comment on "Heat capacity, time constant and sensitivity of Earth's climate system" by S. E. Schwartz, J. Geophys. Res., 113, D15103, doi:10.1029/2007JD009473, 2008.

Langen, P. L. and Alexeev, V. A.: Estimating $2 \times \mathrm{CO}_{2}$ warming in an aquaplanet GCM using the fluctuation-dissipation theorem, Geophys. Res. Lett., 32, L23708, doi:10.1029/2005GL024136, 2005.

Leith, C. E.: Climate response and fluctuation dissipation, J. Atmos. Sci., 32, 2022-2026, 1975.

Levitus, S., Antonov, J., and Boyer, T.: Warming of the world ocean, 1955-2003, Geophys. Res. Lett., 32, L02604, doi:02610.01029/02004GL021592, 2005.

Majda, A., Abramov, R., and Grote, M.: Information Theory and Stochastics for Multiscale Nonlinear Systems, CRM Monograph Series of Centre de Recherches Mathematiques, Universite de Montreal, Vol. 25, American Mathematical Society, ISBN: 08218-3843-1, 2005.
North, G. R., Bell, R. E., and Hardin, J. W.: Fluctuation dissipation in a general circulation model, Clim. Dynam., 8, 259-264, 1993.

Penland, C. and Sardeshmukh, P. D.: The optimal growth of tropical sea surface temperature anomalies, J. Climate, 8, 1999-2024, 1995.

Scafetta, N.: Comment on "Heat capacity, time constant, and sensitivity of Earth's climate system" by S. Schwartz, J. Geophys. Res.,113, D15104, doi:10.1029/2007JD009586, 2008.

Schwartz, S. E.: Heat capacity, time constant, and sensitivity of Earth's climate system, J. Geophys. Res., 112, D24S05, doi:10.1029/2007JD008746, 2007.

Schwartz, S. E.: Response to Comments on "Heat capacity, time constant, and sensitivity of Earth's climate system", J. Geophys. Res., 113, D15105, doi:10.1029/2007JD009872, 2008.

von Storch, J. S.: On statistical dissipation in GCM-climate, Clim. Dynam., 23, 1-15, 2004. 